\title{
TRACE RESULT FOR SOBOLEV EXTENSION DOMAINS
}

\section{DJAMEL AIT-AKLI*, ABDELKADER MERAKEB}

L2CSP, Mouloud Mammeri university of Tizi-Ouzou, 15000, Tizi-Ouzou, Algeria

${ }^{*}$ Corresponding author: djamel.aitakli@ummto.dz

\begin{abstract}
In this paper, we establish the existence and continuity of a trace operator for functions of the Sobolev space $W^{1, p}(\Omega)$ with $1<p<\infty$ on the boundary of a domain $\Omega$ that has the Sobolev $W^{1, p}$-extension property. First, we prove the existence and the continuity of such an operator when it is applied to the elements of the subspace of the up to boundary smooth functions by using a uniform estimate. The essential ingredients used in the proof of this estimate are Green's representation of a function on a disk as well as Banach's isomorphism theorem. Finally, we conclude the trace result using the density of smooth functions in $W^{1, p}(\Omega)$. The presented proof fully exploits the extensibility hypothesis of the domain $\Omega$. The relevance of the result lies in the existence of extension domains which are not Lipschitz and under this point of view it constitutes a generalization of the usual trace theorem.
\end{abstract}

\section{INTRODUCTION}

The trace operator, when applied to the functions of the Sobolev space $W^{1, p}(\Omega)$ defined on a Lipschitz domain $\Omega$, is a standard notion in the theory of Sobolev spaces. More precisely, it is well established that the trace operator is well defined on the boundary of a Lipschitz domain and, moreover, it is continuous, cf. [1]. The result established in this paper generalizes this fact to the case of a non-Lipschitz domain, namely to $W^{1, p}(\Omega)$-extension domains. The regularity of this class of domains is intermediate between Lipschitz regularity and Jordan regularity.

Received March $7^{\text {th }}, 2021$; accepted April 12 $2^{\text {th }}, 2021$; published $0,2021$.

2010 Mathematics Subject Classification. 46E35, 35J57, 26A16, 46B25.

Key words and phrases. Sobolev spaces; $W^{1, p}$-extension domains; Green representation; trace inequality; density of smooth functions.

(C)2021 Authors retain the copyrights of their papers, and all open access articles are distributed under the terms of the Creative Commons Attribution License. 
The functions of the Sobolev space $W^{1, p}(\Omega)$ defined on such a domain constitute a relevant class of functions in the following sense: there exists domains whose boundary is parameterized by a continuous function that is not Lipschitz but admit the property of $(1, p)$ - extension. Indeed, Maz'ya has constructed, cf. [2], an example of a Jordan domain, $\Omega$, such that the boundary $\partial \Omega$ is not Lipschitz in the neighborhood of at least one of its points and such that the property of $(1, p)-$ extension is only valid for $p<2$. We emphasize that a Lipschitz domain is always an extension domain.

We give, herein, a proof of the existence and the boundedness of a trace operator defined on the Sobolev space $W^{1, p}(\Omega)$ where $\Omega$ is a $W^{1, p}$-extension domain, $1<p<\infty$. The proof that we present in the framework of extension domains fully exploits the domain's extensibility hypothesis.

We start by introducing some preliminary notions as well as the tools and results that are essential to carry out the proof of the main result.

\section{Classical facts and tools}

Let $\Omega \subset \mathbb{R}^{2}$ be a domain. We denote by $\operatorname{Int}(\Omega)$ the topological interior of $\Omega$. Throughout this section, and only otherwise explicitly stated, $p$ is a real number with $1<p<\infty$. The usual Sobolev space $W^{1, p}(\Omega)$ is defined by

$$
W^{1, p}(\Omega):=\left\{u \in L^{p}(\Omega), \quad \nabla u \in L^{p}(\Omega)\right\}
$$

We further assume that $\Omega$ has the property of $W^{1, p}$-extension. Recall that the property of $W^{1, p}$-extension of a domain $\Omega$ means that a Sobolev extension operator can be defined on $W^{1, p}(\Omega)$, we recall its definition:

Definition 2.1. We say that a domain $\Omega \subset \mathbb{R}^{2}$ is a $W^{1, p}$-extension domain, if there is a linear operator $P$ defined by

$$
\begin{aligned}
P: W^{1, p}(\Omega) & \rightarrow W^{1, p}\left(\mathbb{R}^{2}\right) \\
u & \rightarrow P u .
\end{aligned}
$$

such that $P$ is continuous i.e. there exists $c_{\text {ext }}>0$ such that $\forall u \in W^{1, p}(\Omega)$ we have

$$
\|P u\|_{W^{1, p}\left(\mathbb{R}^{2}\right)} \leq c_{e x t}\|u\|_{W^{1, p}(\Omega)}
$$

where $c_{\text {ext }}$ depends only on $\Omega$ and $(P u)(x)=u(x)$ almost everywhere in $\Omega$.

The reader may refer to [2] for a detailed discussion of this class of domains. We recall a density result established in ( [3], p.261). This result is valid in the case where $\Omega$ is an extension domain or more generally a Jordan domain:

Theorem 2.1. Let $\Omega \subset \mathbb{R}^{2}$ be a domain whose boundary is a Jordan curve, then $C^{\infty}(\bar{\Omega})$ is dense in $W^{1, p}(\Omega)$ with $1<p<\infty$. 
We recall the property of continuity of the trace operator, on the Lipschitz (or weakly Lipschitz) boundary of a domain $D \subset \mathbb{R}^{2}$, defined for the Sobolev space $W^{1, p}(D)$, cf. [1]:

Proposition 2.1. Let $D \subset \mathbb{R}^{2}$ be a Lipschitz domain. There exists $C_{D}>0$, depending merely on $D$ and $p$, such that

$$
\|u\|_{W^{1-\frac{1}{p}, p}(\partial \Omega)} \leq C_{D}\|u\|_{W^{1, p}(D)}
$$

for all $u \in W^{1, p}(D)$.

It should be noted that proposition 2.1 does not apply in principle neither to an extension domain nor to the more general Jordan domain given the primordial assumption of the Lipschitz character of the ambient domain $D$.

Let us also recall a trace result for the functions of the Sobolev space $W^{1,1}\left(D_{r}\right)$ where $D_{r} \subset \mathbb{R}^{2}$ is a disk of radius $r>0$, cf. ( [4], estimate 7.1):

Proposition 2.2. For all $u \in W^{1,1}\left(D_{r}\right)$ we have

$$
\int_{\partial D_{r}}|u| d \sigma \leq \frac{2}{r} \int_{D_{r}}|u| \mathrm{d} x+\int_{D_{r}}|\nabla u| \mathrm{d} x
$$

In addition, we recall the integral representation of a function $u \in C^{2}\left(D_{r}\right) \cap C^{0}\left(\partial D_{r}\right)$ on the disk $D_{r}$, cf. [5]. For such a function, this representation writes: for all $x \in \operatorname{Int}\left(D_{r}\right)$ we have

$$
u(x)=\int_{D_{r}} \Delta u(y) G(x, y) \mathrm{d} y+\int_{\partial D_{r}} u(y) K(x, y) \mathrm{d} \sigma(y)
$$

where $G$ is the Green function of the disk, $D_{r}$, associated with the Laplace operator and defined by:

$$
G(x, y):=\Gamma(x-y)+h_{x}(y)
$$

with $\Gamma(x)=\frac{1}{2 \pi} \ln |x|$ being the fundamental solution of the Laplace operator in dimension two and $h_{x}$ is the harmonic function in $y$ that equals $-\Gamma(x-y)$ for $x \in \operatorname{Int}\left(D_{r}\right)$ and $y \in \partial D_{r}$. On the other hand $K(x, y)=\frac{\partial G(x, y)}{\partial \nu_{y}}$ is the Poisson kernel which in the case of the disk is written:

$$
K(x, y)=\frac{r^{2}-|x|^{2}}{2 \omega_{2} r|x-y|^{2}}
$$

where $\omega_{2}$ is the measure of the two-dimensional unit ball. This integral representation is interesting insofar as it makes it possible to point-wisely express a function using its Laplacian and the values it takes on the boundary of the disk $D_{r}$. 


\section{Principal Results}

The main result of this paper is stated in the following theorem, its proof will be given after establishing an auxiliary lemma.

Theorem 3.1. Let $\Omega$ be a domain having the $W^{1, p}$-extension property such that $1<p<\infty$, then we can define a trace operator

$$
\begin{aligned}
T: W^{1, p}(\Omega) & \rightarrow L^{p}(\partial \Omega) \\
u & \rightarrow T u
\end{aligned}
$$

that coincides with the restriction operator on the boundary $\partial \Omega$ for continuous functions. In addition, $T$ is continuous i.e. there exists $c_{t}>0$ independent on $u$ such that for all $u \in W^{1, p}(\Omega)$ we have

$$
\|T u\|_{p, \partial \Omega} \leq c_{t}\|u\|_{W^{1, p}(\Omega)}
$$

where we denote \|\|$_{p, \partial \Omega}$ the usual norm of the Lebesgue space of p-integrable functions.

3.1. Auxiliary lemma. We now state with a proof an auxiliary lemma essential for establishing the main theorem:

Lemma 3.1. Let $1<p<2$ and fix a point $x_{0} \in \mathbb{R}^{2}$. We denote $D_{r_{0}}:=D\left(x_{0}, r_{0}\right) \subset \mathbb{R}^{2}$ the disk with center $x_{0}$ and radius $r_{0}>0$. There exists a constant $c\left(r_{0}, p\right)>0$ which is independent of both $u$ and $x$ such that for all $u \in C^{\infty}\left(\overline{D_{r_{0}}}\right)$

$$
\forall x \in D_{\frac{r_{0}}{2}}, \quad|u(x)| \leq c\left(r_{0}, p\right)\|u\|_{W^{1, p}\left(D_{r_{0}}\right)}
$$

We denote by $|x-y|_{2}$ the Euclidean distance between the points $x$ and $y$.

Proof. let $u \in C^{\infty}\left(\overline{D_{r_{0}}}\right)$. There exists $f \in C^{\infty}\left(D_{r_{0}}\right)$ and $u^{d} \in C^{\infty}\left(\partial D_{r_{0}}\right)$ such that the function $u$ solves the problem

$$
\left\{\begin{array}{l}
-\Delta u(y)=f(y) \quad \text { in } \quad D_{r_{0}} \\
u(y) \quad=u^{d}(y) \quad \text { on } \quad \partial D_{r_{0}}
\end{array}\right.
$$

Let $x \in D_{\frac{r_{0}}{2}}$. By using the integral representation (2.4) applied to the function $u$ at $x$, we have:

$$
u(x)=\int_{D_{r_{0}}} f(y) G(x, y) \mathrm{d} y+\int_{\partial D_{r_{0}}} u^{d}(y) K(x, y) \mathrm{d} \sigma(y) .
$$

Given that $\partial D_{r_{0}}$ is $C^{\infty}$-regular, then the Green's function satisfies $G(x,.) \in W^{1, p}\left(D_{r_{0}}\right)$ for $1<p<2$, cf. ( $[6]$, estimate 1.5). By using the continuity of the linear form associated with $f \in W^{-1, p}(\Omega)$, we obtain 
from estimate (3.4) the following

$$
|u(x)| \leq\left.\|f\|_{W^{-1, p}\left(D_{r_{0}}\right)}|| G(x, .)\right|_{W^{1, p}\left(D_{r_{0}}\right)}+\max _{y \in \partial D_{r_{0}}}|K(x, y)| \int_{\partial D_{r_{0}}}\left|u^{d}(y)\right| \mathrm{d} \sigma(y) .
$$

The last term of this inequality does make sense since $|x-y|_{2} \geq \frac{r_{0}}{2}$ for $x \in D_{\frac{r_{0}}{2}}$ and $y \in \partial D_{r_{0}}$. By applying the trace inequality on the boundary of the disc $D_{r_{0}}$, cf. Estimate (2.3), we get

$$
\begin{aligned}
|u(x)| \leq & \|f\|_{W^{-1, p}\left(D_{r_{0}}\right)}\|G(x, .)\|_{W^{1, p}\left(D_{r_{0}}\right)} \\
& +\max _{y \in \partial D_{r_{0}}}|K(x, y)|\left(\frac{2}{r_{0}}\|u\|_{L^{1}\left(D_{r_{0}}\right)}+\|\nabla u\|_{L^{1}\left(D_{r_{0}}\right)}\right),
\end{aligned}
$$

the Holder inequality then yields

$$
\begin{aligned}
|u(x)| \leq & \|f\|_{W^{-1, p}\left(D_{r_{0}}\right)}|| G(x, .) \|_{W^{1, p}\left(D_{r_{0}}\right)} \\
& +\max _{y \in \partial D_{r_{0}}}|K(x, y)| c_{r_{0}}\left(\|u\|_{p, D_{r_{0}}}+\|\nabla u\|_{p, D_{r_{0}}}\right) .
\end{aligned}
$$

We apply Banach's isomorphism theorem to the continuous bijective operator $L_{1}$ defined by

$$
\begin{aligned}
L_{1}:\left(W^{-1, p}\left(D_{r_{0}}\right),\|\|_{W^{-1, p}\left(D_{r_{0}}\right)}\right) & \rightarrow\left(W_{0}^{1, p}\left(D_{r_{0}}\right),\|\|_{W^{1, p}\left(D_{r_{0}}\right)}\right) \\
f & \rightarrow L(f)=u_{1},
\end{aligned}
$$

with $\Delta u_{1}=f$. We then deduce the existence of a constant $c_{1}^{\prime}$ independent of $f$ such that

$$
\begin{aligned}
|u(x)| \leq & c_{1}^{\prime}\left\|u_{1}\right\|_{W_{0}^{1, p}\left(D_{r_{0}}\right)}|| G(x, .) \|_{W^{1, p}\left(D_{r_{0}}\right)} \\
& +\max _{y \in \partial D_{r_{0}}}|K(x, y)| c_{r_{0}}|| u \|_{W^{1, p}\left(D_{r_{0}}\right)}
\end{aligned}
$$

with $u_{1}=u-u_{2}$ and $u_{2}$ solves the problem

$$
\left\{\begin{array}{l}
-\Delta u_{2}=0 \quad \text { in } \quad D_{r_{0}}, \\
u_{2}=u^{d} \text { on } \partial D_{r_{0}} .
\end{array}\right.
$$

By using the Poincaré inequality, the estimate (3.5) then becomes

$$
\begin{aligned}
|u(x)| \leq & c_{1}\left(\|\nabla u\|_{p, D_{r_{0}}}+\left\|\nabla u_{2}\right\|_{p, D_{r_{0}}}\right)\|G(x, .)\|_{W^{1, p}\left(D_{r_{0}}\right)} \\
& +\max _{y \in \partial D_{r_{0}}}|K(x, y)| c_{r_{0}}\|u\|_{W^{1, p}\left(D_{r_{0}}\right)} .
\end{aligned}
$$

Using Proposition 2.1, we can apply the isomorphism theorem to the operator associated with the problem (3.6) to establish the existence of a constant $c_{2}$ such that

$$
\left\|\nabla u_{2}\right\|_{p, D_{r_{0}}} \leq c_{2}\left\|u_{2}\right\|_{W^{1-\frac{1}{p}, p}\left(\partial D_{r_{0}}\right)}
$$


where $c_{2}$ is independent of $u_{2}$ and $\Delta u_{2}=0$. The estimate (3.7) becomes

$$
\begin{aligned}
|u(x)| \leq & c_{1}\left(\|\nabla u\|_{p, D_{r_{0}}}+c_{2}\|u\|_{W^{1-\frac{1}{p}, p}\left(\partial D_{r_{0}}\right)}\right)\|G(x, .)\|_{W^{1, p}\left(D_{r_{0}}\right)} \\
& +\max _{y \in \partial D_{r_{0}}}|K(x, y)| c_{r_{0}}\left(\|u\|_{p, D_{r_{0}}}+\|\nabla u\|_{p, D_{r_{0}}}\right) .
\end{aligned}
$$

Applying the trace theorem in $W^{1, p}\left(D_{r_{0}}\right)$, cf. Proposition 2.1, allows to infer the existence of a constant $c_{3}>0$ such that

$$
\begin{aligned}
|u(x)| \leq & c_{1}\left(\|\nabla u\|_{p, D_{r_{0}}}+c_{3}\left(\|u\|_{p, D_{r_{0}}}+\|\nabla u\|_{p, D_{r_{0}}}\right)\right)\|G(x, .)\|_{W^{1, p}\left(D_{r_{0}}\right)} \\
& +\max _{y \in \partial D_{r_{0}}}|K(x, y)| c_{r_{0}}\|u\|_{W^{1, p}\left(D_{r_{0}}\right)},
\end{aligned}
$$

then we infer that there exists a constant $c\left(r_{0}, p\right)$ which is independent of both $u$ and $x$ such that

$$
\forall x \in D_{\frac{r_{0}}{2}}, \quad|u(x)| \leq c\left(r_{0}, p\right)\|u\|_{W^{1, p}\left(D_{r_{0}}\right)} .
$$

This last estimate is obtained by using the fact that $\|G(x, .)\|_{W^{1, p}\left(D_{r_{0}}\right)}$ is uniformly bounded for $x \in$ $D_{\frac{r_{0}}{2}}$.

We now present a proof of Theorem 3.1:

\subsection{Proof of the main result.}

Proof. Let $u \in C^{\infty}(\bar{\Omega})$. The function $u$ is obviously Lipschitz on $\bar{\Omega}$. If we denote $L$ the Lipschitz constant of the function $x \rightarrow u(x)$ relatively to the domain $\Omega$, then for all $x \in \partial \Omega$ and all $y \in \operatorname{Int}(\Omega)$ we have

$$
|u(x)-u(y)| \leq L|x-y|_{2},
$$

which immediately implies

$$
|u(x)| \leq L|x-y|_{2}+|u(y)|
$$

for all $x \in \partial \Omega$ and all $y \in \operatorname{Int}(\Omega)$. Let $x \in \partial \Omega$ be fixed and let $\left(y_{\delta}\right)_{\delta>0}$ be a sequence of point such that $y_{\delta} \in \operatorname{Int}(\Omega)$ and $\left|y_{\delta}-x\right|_{2} \rightarrow 0$ when $\delta \rightarrow 0$. Let's fix $\delta>0$. The estimate (3.8) yields

$$
|u(x)| \leq L\left|x-y_{\delta}\right|_{2}+\left|u\left(y_{\delta}\right)\right|
$$

for all $\delta>0$. Pose $\tilde{u}=\mathrm{P} u$ with $\mathrm{P}$ being an extension operator defined on $W^{1, p}(\Omega)$ whose range is $W^{1, p}\left(\mathbb{R}^{2}\right) \subset W^{1, p}(\Omega)$, cf. Definition 2.1. Such an extension operator is well defined.

It should be noted that the function $\tilde{u}$ is not necessarily continuous over $\mathbb{R}^{2}-\Omega$ i.e. it does not have a representative continuous function. Indeed, this is due to the fact that $1-\frac{2}{p}<0$ for $1<p<2$; therefore the classical Sobolev embedding into Holder spaces doesn't apply in this case, namely when $1<p<\infty$.

Since $\Omega$ is bounded, there exists $r_{0}>0$ and $x_{0} \in \Omega$ such that if we denote $D_{r_{0}}$ the disk with center $x_{0}$ and radius $r_{0}$ then we have $\Omega \subset D_{r_{0}}$. The set, $C^{\infty}\left(\overline{D_{2 r_{0}}}\right)$, of smooth functions up to the boundary defined 
on the Lipschitz domain $\overline{D_{2 r_{0}}} \subset \mathbb{R}^{2}$ being dense in $W^{1, p}\left(D_{2 r_{0}}\right)$, cf. Theorem 2.1, therefore there exists a sequence $\left(v_{n}\right)_{n}, v_{n} \in C^{\infty}\left(\overline{D_{2 r_{0}}}\right)$, such that

$$
\left\|v_{n}-\tilde{u}\right\|_{W^{1, p}\left(D_{2 r_{0}}\right)} \rightarrow 0 \quad n \rightarrow \infty
$$

The estimate (3.10) implies that there exists a subsequence $(\phi(n))_{n}$ such that $v_{\phi(n)}(x) \rightarrow \tilde{u}(x)$ a.e. in $D_{2 r_{0}}$. As $v_{n}$ and $\tilde{u}$ are continuous in $\Omega$, this almost everywhere convergence is valid everywhere in $\Omega$ i.e.

$$
\forall y \in \Omega, \quad v_{\phi(n)}(y) \rightarrow \tilde{u}(y), \quad \text { when } n \rightarrow \infty .
$$

Using (3.9) and (3.11) we get: $\forall \delta>0$ and $\forall \epsilon>0, \exists n\left(\epsilon, y_{\delta}\right)>0$ such that:

$$
|u(x)| \leq L\left|x-y_{\delta}\right|_{2}+\left|v_{\phi\left(n\left(\epsilon, y_{\delta}\right)\right)}\left(y_{\delta}\right)\right|+\epsilon,
$$

where $\epsilon>0$ is intended to tend towards zero and $\left(n\left(\epsilon, y_{\delta}\right)\right)_{\epsilon>0}$ is a sequence of integers which tends to $+\infty$ when $\epsilon \rightarrow 0$ for all $\delta>0$. On the other hand, the formula (3.12) and the regularity of $v_{\phi\left(n\left(\epsilon, y_{\delta}\right)\right)}$ allow to write

$$
|u(x)| \leq L\left|x-y_{\delta}\right|_{2}+\left|v_{\phi\left(n\left(\epsilon, y_{\delta}\right)\right)}\right|_{\infty, \overline{D_{r_{0}}}}+\epsilon,
$$

for all $\delta>0$. By applying the lemma 3.1 in the disk $D_{2 r_{0}}$ with center $x_{0}$, we have

$$
|u(x)| \leq L\left|x-y_{\delta}\right|_{2}+c\left(r_{0}, p\right)\left\|v_{\phi\left(n\left(\epsilon, y_{\delta}\right)\right.}\right\|_{W^{1, p}\left(D_{2 r_{0}}\right)}+\epsilon
$$

where $c\left(r_{0}, p\right)$ is the constant appearing in the estimate (3.2). The estimate (3.13) is valid for all $\epsilon>0$ independently of $\delta$. So, passing to the limit $\epsilon \rightarrow 0$ and using Estimate (3.10), we obtain

$$
|u(x)| \leq L\left|x-y_{\delta}\right|_{2}+\left.c\left(r_{0}, p\right)|| \tilde{u}\right|_{W^{1, p}\left(D_{2 r_{0}}\right)},
$$

for all $\delta>0$. Given that all the elements involved in the estimate (3.14) are independent of $\delta$ then by letting $\delta \rightarrow 0$ we find

$$
|u(x)| \leq c\left(r_{0}, p\right)\|\tilde{u}\|_{W^{1, p}\left(D_{2 r_{0}}\right)} .
$$

So the estimate (3.15) yields us

$$
|u(x)| \leq c\left(r_{0}, p\right)|| P u \|_{W^{1, p}\left(\mathbb{R}^{2}\right)} .
$$

Using (2.1), the estimate (3.16) in turn gives

$$
|u(x)| \leq c\left(r_{0}, p\right) c_{e x t}|| u \|_{W^{1, p}(\Omega)} .
$$

Note that the estimate (3.17) is valid for all $x \in \Gamma:=\partial \Omega$ independently of the quantities present at the right side of this inequality. 
Now, let us denote $t \in[a, b] \rightarrow x(t)=(t, \gamma(t))$ the parametric function representation of the curve $\Gamma$. So, according to (3.17), we have:

$$
|u(t, \gamma(t))|^{p} \leq\left(c\left(r_{0}, p\right) c_{e x t}\right)^{p}\|u\|_{W^{1, p}(\Omega)}^{p},
$$

for all $t \in[a, b]$. By definition of the line integral, we have

$$
\int_{\Gamma}|u|^{p} \mathrm{ds}:=\int_{a}^{b}|u \circ \gamma|^{p} \mathrm{~d} s_{\gamma}
$$

Thus, by integrating the two sides of (3.18) with respect to the curvilinear abscissa $\mathrm{d} s_{\gamma}$ and using (3.19), we find

$$
\int_{\Gamma}|u|^{p} \mathrm{ds} \leq\left(c\left(r_{0}, p\right) c_{e x t}\right)^{p}\|u\|_{W^{1, p}(\Omega)}^{p} \int_{a}^{b} 1 \mathrm{~d} s_{\gamma}
$$

it yields

$$
\int_{\partial \Omega}|u|^{p} \mathrm{ds} \leq\left(c\left(r_{0}, p\right) c_{e x t}\right)^{p}\|u\|_{W^{1, p}(\Omega)}^{p}|\partial \Omega| .
$$

Set $c_{t}:=\left[\left(c\left(r_{0}, p\right) c_{e x t}\right)^{p}|\partial \Omega|\right]^{\frac{1}{p}}$. This constant does not depend on $u$ but merely on $\Omega$ and on the exponent $p$. From (3.20), we have for all $u \in C^{\infty}(\bar{\Omega})$

$$
\|u\|_{p, \partial \Omega} \leq c_{t}\|u\|_{W^{1, p}(\Omega)}
$$

Currently, we conclude the estimate (3.1). According to Theorem 2.1, for any $u \in W^{1, p}(\Omega)$ there exists $\left(u_{l}\right)_{n \in \mathbb{N}}, u_{l} \in C^{\infty}(\bar{\Omega})$, such that

$$
\left\|u_{l}-u\right\|_{W^{1, p}(\Omega)} \rightarrow 0
$$

Thus, by applying (3.21) to the elements of the sequence $\left(u_{l}\right)_{l}$, we have for all $l \in \mathbb{N}$ the following estimate:

$$
\left\|u_{l}\right\|_{p, \partial \Omega} \leq c_{t}|| u_{l} \|_{W^{1, p}(\Omega)} .
$$

Since $\left(u_{l}\right)_{l}$ is a Cauchy sequence in the normed space $W^{1, p}(\Omega)$ then estimate (3.22) implies that it is also a Cauchy sequence in the normed space $L^{p}(\partial \Omega)$. But $L^{p}(\partial \Omega)$ is complete, then there exists $u^{*} \in L^{p}(\partial \Omega)$ such that

$$
\left\|u^{*}\right\|_{p, \partial \Omega} \leq c_{t}\|u\|_{W^{1, p}(\Omega)} .
$$

Finally, by setting $\mathrm{T} u:=u^{*}$, we have

$$
\|\mathrm{T} u\|_{p, \partial \Omega} \leq c_{t}\|u\|_{W^{1, p}(\Omega)}
$$


for all $u \in W^{1, p}(\Omega)$. This defines a continuous trace operator on the space $L^{p}(\partial \Omega)$ on the boundary $\partial \Omega$

$$
\begin{aligned}
T: W^{1, p}(\Omega) & \rightarrow L^{p}(\partial \Omega) \\
u & \rightarrow T u
\end{aligned}
$$

Remark 3.1. The relevance of the established result lies in the fact that it is valid for functions of the space $W^{1, p}(\Omega)$ which are not necessarily continuous for $1<p<2$. The existence of the trace for this category of functions is not obvious. The example of the domain constructed by Mazya in [2] illustrates perfectly not only the relevance but also the generality which results from the fact that we have considered the domain $\Omega$ having the $W^{1, p}$-extension property.

Acknowledgment. The authors would like to thank very much the anonymous referees for reviewing the article as well as the editorial staff for their support during the submission process.

Conflicts of Interest: The author(s) declare that there are no conflicts of interest regarding the publication of this paper.

\section{REFERENCES}

[1] E. Gagliardo, Caratterizzazioni delle tracce sulla frontiera relative ad alcune classi di funzioni in $\mathrm{n}$ variabili, Ren. Sem. Mat. Univ. Padova. 27 (1957), 284-305.

[2] V.G. Maz'ya, Extension of functions from Sobolev spaces, J. Math. Sci. 22 (1983), 1851-1855.

[3] J.L. Lewis, Approximation of Sobolev functions in Jordan domains, Ark. Mat. 25 (1987), 255-264.

[4] G. Auchmuty, Sharp boundary trace inequalities, Proc. R. Soc. Edinburgh Sect. A: Math. 144 (2014), 1-12.

[5] L.C. Evans, Partial Differential Equations, Graduate Studies in Mathematics, Americam Mathematical Society, Providence, 1998.

[6] D. Mitrea, I. Mitrea, On the Regularity of Green Functions in Lipschitz Domains, Commun. Part. Differ. Equ. 36 (2010), $304-327$. 